\title{
Separability of Multipartite Quantum States with Strong Positive Partial Transpose
}

\author{
Lilong Qian* \\ Department of Mathematics, National University of Singapore, 119076, Singapore
}

(Dated: May 2, 2018)

\begin{abstract}
We generalize the definition of strong positive partial transpose (SPPT) to the multipartite system. The tripartite case was first considered by X.-Y. Yu and H. Zhao [ Int. J. Theor. Phys., 54, 292, (2015)]. In this extension, unfortunately, desired properties such as the PPT of SPPT states and the separability of super and pure SPPT states are not preserved. In contrast, this paper provides an alternative generalization to multipartite cases with these properties preserved. We also provide sufficient conditions for the separability of SPPT states.

PACS numbers: 03.65.Ud, 03.67.Mn
\end{abstract}

\section{INTRODUCTION}

Entanglement lies at the heart of quantum computation and information theory, which is the resource of most applications in quantum information processing tasks. Since 1935, when the necessarily nonlocal nature of quantum mechanics was first highlighted by Einstein, Podolsky and Rosen (EPR) [1], quantum entanglement has become a major quantum phenomenon which requires further understanding. One of the fundamental problems about quantum entanglement is the separability problem i.e. to check whether a given quantum state is separable or entangled. Given a density matrix $\rho$ in a quantum bipartite system $A: B, \rho$ is said to be separable if it can be written as a convex combination of product states [2], i.e. $\rho$ is separable if

$$
\rho=\sum_{i} p_{i} \rho_{i}^{A} \otimes \rho_{i}^{B}, \sum_{i} p_{1}=1, p_{i} \geqslant 0
$$

where $\rho_{i}^{A}$ and $\rho_{i}^{B}$ are the density matrices in subsystems $A$ and $B$ respectively. A quantum state is said to be quantum entangled if the density matrix does not possess a decomposition of the form as Eq. (1). Unlike separable states, entangled states cannot be obtained by preparing their subsystems [3].

Despite remarkable efforts over recent years, the operational necessary and sufficient condition for the separability still remains unknown in general. It has been found that the separable problem is NP-HARD even for the bipartite system [4].

While it is hard to solve this problem in general, there are plenty of practical criteria which enable us to detect entanglement for some sub-classes. One of the most famous criteria is called positive partial transpose (PPT) or Peres-Horodecki criterion [3]. It tells that if a state $\rho$ is separable, then its partial transposed state $\rho^{T_{A}}=(\mathrm{T} \otimes \mathbb{1}) \rho$ remains positive. Using positive maps, Horodecki et al. [5] showed that Peres-Horodecki criterion is also sufficient for $2 \otimes 2$ and $2 \otimes 3$ systems. It is, however,

* Electronic adress: qian.lilong@u.nus.edu not true for higher dimensional spaces. Woronowicz [6] constructed a counterexample of a $2 \otimes 4$ entangled PPT state. See more entangle PPT states in Refs. [7-9]. Utilizing matrix analysis, Kraus et al. [10] showed that any $M \otimes N$ PPT state of rank $N$ is separable. Moreover, some generalized results are proposed in Refs. [11-13]. Since any $M \otimes N$ state of rank less than $N$ is distillable [14], it suffices to consider these state whose rank is greater than its local ranks.

A subclass of PPT states, namely strong PPT (SPPT) states, were first considered by Chruściński et al. [15]. These states have a "strong PPT property". Based on several examples, it was conjectured that SPPT states are separable. Unfortunately, this conclusion fails for $M \otimes N$ PPT states when $N M \geq 9$. Actually, all $2 \otimes 4$ SPPT states are separable [16]. But, there exists a $2 \otimes 5$ SPPT which is entangled [16]. The separability of SPPT states become more complex in high dimensional spaces. The SPPT states encompass many previously known separable PPT states such as rank $N$ states of $2 \otimes N$ system. Moreover, it is proved that SPPT states can be used to witness quantum discord (QD) in $2 \otimes N$ systems [17]. In addition, Bylicka et al. [18] constructed a special class of SPPT states, which were called super strong SPPT (SSPPT) states. In Ref. [19], the decomposition of SSPPT states was considered in both finite and infinite dimensional systems.

In a recent paper[20], the idea of SPPT states was generalized to the tripartite system $A_{1}: A_{2}: A_{3}$. However, these states are essentially bipartite SPPT with respect to the bi-partition $A_{1} A_{2}: A_{3}$. As a result, some good properties may be lost in the tripartite sense. For instance, the SPPT cannot guarantee PPT, which is one of the most important features for SPPT states in the bipartite system. Also, the super SPPT cannot guarantee the separability in general. Therefore, it would be especially interesting to find a more appropriate generalization to tripartite or even multipartite systems. The purpose of this paper is to provide an alternative definition of SPPT states in the $n$-particle system. We begin with the simplest $2 \otimes 2 \otimes N$ case, and eventually, extend to general many-body system.

The remainder of this paper is organized as follows. In Section II, we present some preliminaries about the sep- 
arability problem of the SPPT states. In Section III, we recall the definition of tripartite SPPT states in Ref. [20]. We showed that the defined SPPT and SSPPT cannot inherit many good properties as those in the bipartite systems. In Section IV, we provide a new idea to define the SPPT and SSPPT state in $2 \otimes 2 \otimes N$ system. We extend this concept to $N_{1} \otimes N_{2} \otimes N_{3}$ case. Finally, we show the idea to the arbitrary multipartite system. In Section V, we propose some sufficient conditions for separability of SPPT states. Some concluding remarks are given in Section VI.

\section{PRELIMINARIES}

We start this section with a formal definition of separability. Consider a $d$-particle state belonging to a Hilbert space $\mathcal{H}$. Denote by $A_{1}, A_{2}, \cdots, A_{d}$ the subsystems respectively. Each subsystem is a Hilbert space $\mathcal{H}_{i}$ with dimension $N_{i}$. By the postulate for composition of system in quantum computation thery, we have $\mathcal{H}=\otimes_{i=1}^{d} \mathcal{H}_{i}$ and $\operatorname{dim}(\mathcal{H})=\prod_{i=1}^{d} N_{i}$.

To make more concise, we use vector based indexes in this paper. Let $\mathcal{I}$ be the set consisting of d-tuples $\left(i_{1}, i_{2}, \ldots, i_{d}\right), 1 \leqslant i_{k} \leqslant N_{k}$. Given a index $\alpha=$ $\left(i_{1}, i_{2}, \ldots, i_{d}\right) \in \mathcal{I}$, then $x_{\alpha}$ represents $x_{i_{1}, i_{2}, \ldots, i_{d}}$. Furthermore, we also assign an order for those indexes. Let

$$
n(\alpha)=\prod_{k=1}^{d-1}\left(\prod_{l=k+1}^{d} N_{k}\right)\left(\alpha_{k}-1\right)+\alpha_{d}
$$

for all $\alpha \in \mathcal{I}$. Then we say $\alpha \leqslant \beta$ if $n(\alpha) \leqslant n(\beta)$ for $\alpha, \beta \in \mathcal{I}$. Moreover, $|\alpha\rangle$ represents the product vector $\left|i_{1}, i_{2}, \ldots, i_{d}\right\rangle$.

Hence a density matrix acting on the space $\mathcal{H}$ can be represented as

$$
\rho=\sum_{\alpha, \beta \leqslant \alpha_{0}} \rho_{\alpha, \beta}|\alpha\rangle\langle\beta|,
$$

where $\alpha, \beta \in \mathcal{I}, \alpha_{0}=\left(N_{1}, N_{2}, \ldots, N_{d}\right)$.

Now we recall the definition of separability of a quantum state. A density matrix $\rho$ in $\mathcal{H}$ is said to be separable if it can be written as

$$
\rho=\sum_{i=1}^{L} \lambda_{i}\left|x_{i}\right\rangle\left\langle x_{i}\right|
$$

where $\sum_{i}^{L} \lambda_{i}=1, \lambda_{i} \geqslant 0$ and each $\left|x_{i}\right\rangle$ is a pure product vector in the space $\mathcal{H}$.

Peres-Horodecki criterion plays a crucial role in the separability problem, which is based on the partial transpose. Therefore it would be necessarily to introduce the notations of partial transposes ahead of time. Let $\rho$ be a given state in the composite system $A_{1}: A_{2}$. Denote by $\mathrm{T}$ the usual transpose operator. Then the composite operators $(\mathbb{1} \otimes T)$ and $(T \otimes \mathbb{1})$ are called the partial transpose operators. Furthermore, the partial transposed density matrices are denoted by $\rho^{\mathrm{T}_{2}}=(\mathbb{1} \otimes \mathrm{T}) \rho$ and $\rho^{\mathrm{T}_{1}}=$
$(\mathrm{T} \otimes \mathbb{1}) \rho$. For general $d$-particle system $A_{1}: A_{2} \cdots: A_{d}$, we denote by $\mathrm{T}_{i}(i=1,2, \ldots, d)$ the partial transpose with respect to $i$-th subsystem respectively. The corresponding partial transposed state is denoted by $\rho^{\mathrm{T}_{i}}$. Generally, given an index set $I=\left\{i_{1}, i_{2}, \ldots, i_{k}\right\}, \mathrm{T}_{I}$ denotes the partial transpose with respect to the subsystems in $I$, that is

$$
\mathrm{\top}_{I}=o_{k \in I} \mathrm{\top}_{k}
$$

In the $d$-body system, we introduce a special partial transpose,

$$
\Gamma_{k}=\underbrace{\mathrm{T} \otimes \mathrm{T} \otimes \cdots \otimes \mathrm{T}}_{k \mathrm{~T}} \otimes \underbrace{\mathbb{1} \otimes \cdots \otimes \mathbb{1}}_{(d-k) \mathrm{T}},
$$

which will be used in the following sections.

PPT criterion tells that if $\rho$ is separable, then

$$
\mathrm{T}_{I} \cdot \rho \geqslant 0,
$$

for any $I \subset\{1,2, \ldots, d\}$.

Now we recall the definition of SPPT in the bipartite system.

Consider a density matrix $\rho$ in $N_{1} \otimes N_{2}$ system with a block Cholesky decomposition $\rho=X^{\dagger} X$,

$$
\begin{aligned}
X & =\left(\begin{array}{ccc}
X_{11} & \cdots & X_{1 N_{1}} \\
\vdots & \ddots & \vdots \\
X_{N_{1} 1} & \cdots & X_{N_{1} N_{1}}
\end{array}\right) \\
& =\left(\begin{array}{ccc}
S_{11} X_{1} & \cdots & S_{1 N_{1}} X_{1} \\
\vdots & \ddots & \vdots \\
S_{N_{1} 1} X_{N_{1}} & \cdots & S_{N_{1} N_{1}} X_{N_{1}}
\end{array}\right) \\
& =\left(\begin{array}{ccc}
X_{1} & \cdots & S_{1 N_{1}} X_{1} \\
\vdots & \ddots & \vdots \\
0 & \cdots & X_{N_{1}}
\end{array}\right),
\end{aligned}
$$

where $S_{i j}$ and $X_{i}$ are both $N_{1} \times N_{1}$ matrix with

$$
S_{i j}= \begin{cases}\mathbb{1}_{N_{2}}, & \text { if } i=j \\ 0, & \text { if } i>j\end{cases}
$$

In this paper, $\mathbb{1}_{n}$ denotes the identity operator acting on the space $\mathbb{C}^{n}$.

Definition 1. Let $\rho$ be a density matrix in $N_{1} \otimes N_{2}$ system. And $\rho=X^{\dagger} X$, where $X$ has the form as Eq. (6). Then $\rho$ is said to be SPPT if

$$
\rho^{\mathrm{T}_{1}}=Y^{\dagger} Y
$$

with

$$
Y=\left(S_{i j}^{\dagger} X_{i}\right)
$$

or equivalently,

$$
\sum_{k=1}^{N_{1}} X_{k}^{\dagger}\left[S_{k j}^{\dagger}, S_{k i}\right] X_{k}=0,1 \leqslant i \leqslant j \leqslant N_{1} .
$$


Here the commutator $[A, B]$ is defined by $[A, B]=$ $A B-B A$.

In particular, Eq. (8) is naturally satisfied if

$$
\left[S_{k j}^{\dagger}, S_{k i}\right]=0,1 \leqslant i \leqslant j \leqslant N_{1} .
$$

We call this subclass of SPPT states super SPPT (SSPPT) states [15]. It was proved that every SSPPT state is separable [18].

\section{PREVIOUS DEFINITION OF TRIPARTITE SPPT STATES}

In this section, we will first introduce the definition of tripartite SPPT states in Ref. [20]. After that, we will show that the SPPT states will not preserve some good properties as that in the bipartite system. For example, the SPPT state may not be PPT. Besides, pure or super SPPT states may not be separable.

Suppose that $\rho$ is a density matrix in the tripartite system $A_{1}: A_{2}: A_{3}$, with a decomposition $\rho=X^{\dagger} X$. Under the bi-partition $A_{1}: A_{2} A_{3}, X$ can be written as an $N_{1} \times N_{1}$ block matrix:

$$
X=\left(Z_{i j}\right), Z_{i j} \in M_{N_{2} N_{3}} .
$$

Again, each $Z_{i j}$ can be written as a $N_{2} \times N_{2}$ block matrix:

$$
Z_{i j}=\left(Z_{i j, k l}\right)=\left(S_{i j k l} X_{i k}\right),
$$

where

$$
S_{i j k l}= \begin{cases}\mathbb{1}_{N_{3}}, & (i, k)=(j, l) \\ 0, & (i, k)>(j, l)\end{cases}
$$

Note that the order $(i, k) \leqslant(j, l)$ means $(i-1) N_{1}+k$ $\leqslant(j-1) N_{1}+l$, which is the order we have defined in the previous section. Let $\alpha=(i, k), \beta=(j, l)$, then the decomposition can be written in a conciser form,

$$
\rho=X^{\dagger} X, X=\left(S_{\alpha \beta} X_{\alpha}\right) .
$$

Recall the definition of SPPT state in Ref. [20]:

Definition 2. Let $\rho=X^{\dagger} X$ be a density matrix in the tripartite system $A_{1}: A_{2}: A_{3}$ with $X$ being the form as Eq. (13). Then $\rho$ is said to be SPPT if

$$
\rho^{\mathrm{T}_{12}}=Y^{\dagger} Y
$$

where

$$
Y=\left(S_{\alpha \beta}^{\dagger} X_{\alpha}\right)
$$

Note that in the above definition, the condition (14) is equivalent to

$$
\sum_{\alpha \leqslant \gamma_{1}} X_{\alpha}^{\dagger}\left[S_{\alpha, \beta}, S_{\alpha, \beta^{\prime}}^{\dagger}\right] X_{\alpha}=0
$$

where $\gamma_{0}=(1,1), \gamma_{1}=\left(N_{1}, N_{2}\right)$, and $\gamma_{0} \leqslant \beta \leqslant \beta^{\prime} \leqslant \gamma_{1}$.

Similarly, super SPPT (SSPPT) are also defined for tripartite system in Ref. [20].

Definition 3. Let $\rho$ be the SPPT state with a decomposition of form as Eq. (13), then $\rho$ is SSPPT if

$$
\left[S_{\alpha, \beta}, S_{\alpha, \beta^{\prime}}^{\dagger}\right]=0, \gamma_{0} \leqslant \alpha \leqslant \beta \leqslant \beta^{\prime} \leqslant \gamma_{1}
$$

Note that $\alpha$ refers to the $n(\alpha)$-th row and $\beta$ refers to the $n(\beta)$-th column. Therefore, if we reorder the 2 -tuple $\alpha, \beta$ by a single index $n(\alpha)$ and $n(\beta)$ respectively, then the definition of SPPT will be identical to that in the bipartite system. This implies that some properties of SPPT states may hold only under the bi-partition $A_{1} A_{2}$ : $A_{3}$.

PPT, as is well-known, is one of the most important features for SPPT states. However, the tripartite SPPT states defined here may lose this property. Here we construct an example show this defect.

Let

$$
\rho=v^{\dagger} v, v=(1,0,0,0,0,0,1,0)
$$

be the density matrix in $2 \otimes 2 \otimes 2$ system and

$$
X_{11}=\left(\begin{array}{ll}
1 & 0 \\
0 & 0
\end{array}\right) \text {. }
$$

Then $\rho$ can be written as

$$
\rho=X^{\dagger} X, X=\left(\begin{array}{cccc}
X_{11} & 0 & 0 & X_{11} \\
0 & 0 & 0 & 0 \\
0 & 0 & 0 & 0 \\
0 & 0 & 0 & 0
\end{array}\right)
$$

Put

$$
S_{\alpha, \beta}= \begin{cases}\mathbb{1}, & \alpha=\beta=(1,1) \\ 0, & \text { otherwise }\end{cases}
$$

Therefore $\rho$ satisfies the condition of SSPPT. However, $\rho$ is not a separable state. Since any pure state is separable if and only if it is PPT, $\rho$ is consequently not a PPT state as well. This example illustrates that SPPT cannot guarantee PPT with this definition. Moreover, unlike the bipartite case, super and pure SPPT states may not be separable.

Recall the Theorem 1 of Ref. [20]:

Theorem 1. If $\rho$ us a super SPPT state in $N_{1} \otimes N_{2} \otimes N_{3}$ systems, then $\rho$ is bi-separable. 
It pointed out in the proof that tripartite SSPPT state is bi-separable with respect to any of the bi-partitions $A_{1} A_{2}: A_{3}, A_{1}: A_{2} A_{3}$ and $A_{1} A_{3}: A_{2}$. However this may not be true. Again, consider the example $\rho=v^{\dagger} v$. It is super SPPT by definition. But it is not separable under the bi-partition $A_{1}: A_{2} A_{3}$ and $A_{1} A_{3}: A_{2}$.

In the next section, we will define our SPPT and SSPPT states in another way. It turns out many good properties will be preserved.

\section{SPPT STATES IN MULTIPARTITE CASE}

In this section, we provide a new idea to define the tripartite SPPT states. We begin with the simplest case $2 \otimes 2 \otimes N$, then we extend the idea to general $N_{1} \otimes N_{2} \otimes N_{3}$ tripartite system. Lastly, we give the definition of SPPT in the arbitrary multipartite system. Correspondingly, the SSPPT states are also defined, which turn out to be separable. In addition, we give some examples of SPPT states, which may be helpful to shed new lights on understanding the structure of PPT states in multipartite system.

\section{A. SPPT states in $2 \otimes 2 \otimes N$ system}

We begin with considering the simplest case when $\rho$ is a density matrix in $2 \otimes 2 \otimes N$ system.

Let

$$
X=\left(\begin{array}{cc}
X_{1} & S X_{1} \\
0 & X_{2}
\end{array}\right), Z=\left(\begin{array}{cc}
X_{1} & S^{\dagger} X_{1} \\
0 & X_{2}
\end{array}\right)
$$

where

$$
X_{1}=\left(\begin{array}{cc}
Y_{11} & T_{1} Y_{11} \\
0 & Y_{12}
\end{array}\right), X_{2}=\left(\begin{array}{cc}
Y_{21} & T_{2} Y_{21} \\
0 & Y_{22}
\end{array}\right) .
$$

Here $S$ is a diagonal block matrix

$$
S=\left(\begin{array}{cc}
S_{1} & 0 \\
0 & S_{2}
\end{array}\right)
$$

Hence, $X$ can be written as a $4 \times 4$ block matrix with each block being a $N \times N$ matrix

$$
X=\left(\begin{array}{cccc}
Y_{11} & T_{1} Y_{11} & S_{1} Y_{11} & S_{1} T_{1} Y_{11} \\
0 & Y_{12} & 0 & S_{2} Y_{12} \\
0 & 0 & Y_{21} & T_{2} Y_{21} \\
0 & 0 & 0 & Y_{22}
\end{array}\right)
$$

Let

$$
W=\left(\begin{array}{cccc}
Y_{11} & T_{1}^{\dagger} Y_{11} & S_{1}^{\dagger} Y_{11} & \left(S_{1} T_{1}\right)^{\dagger} Y_{11} \\
0 & Y_{12} & 0 & S_{2}^{\dagger} Y_{12} \\
0 & 0 & Y_{21} & T_{2}^{\dagger} Y_{21} \\
0 & 0 & 0 & Y_{22}
\end{array}\right)
$$

Now we are ready to define SPPT states in the $2 \otimes 2 \otimes N$ system with the above notations.
Definition 4. Let $\rho=X^{\dagger} X$ be a density matrix in $2 \otimes 2$ $\otimes N$ system where $X$ has the form as Eq. (22). Then $\rho$ is said to be SPPT if

$$
\rho^{\mathrm{T}_{1}}=Z^{\dagger} Z
$$

and

$$
\rho^{\top_{12}}=W^{\dagger} W
$$

Alternatively, the above two conditions in the definition of SPPT can be reformulated as

$$
\begin{cases}Y_{11}^{\dagger}\left[T_{1}, T_{1}^{\dagger}\right] Y_{11} & =0, \\ Y_{11}^{\dagger}\left[S_{1}, S_{1}^{\dagger}\right] Y_{11} & =0, \\ Y_{11}^{\dagger}\left[T_{1}, S_{1}^{\dagger}\right] Y_{11} & =0, \\ Y_{11}^{\dagger}\left[S_{1}, T_{1}^{\dagger} S_{1}^{\dagger}\right] Y_{11} & =0, \\ Y_{11}^{\dagger}\left[T_{1}, T_{1}^{\dagger} S_{1}^{\dagger}\right] Y_{11} & =0, \\ Y_{11}^{\dagger}\left[S_{1} T_{1},\left(S_{1} T_{1}\right)^{\dagger}\right] Y_{11} & \\ \quad-Y_{12}^{\dagger}\left[S_{2}^{\dagger}, S_{2}\right] Y_{12}+Y_{21}^{\dagger}\left[T_{2}^{\dagger}, T_{2}\right] Y_{21} & =0 \\ Y_{11}^{\dagger}\left[S_{1}, S_{1}^{\dagger}\right] T_{1} Y_{12} & =0 \\ Y_{12}^{\dagger} T_{1}^{\dagger}\left[S_{2}, S_{2}^{\dagger}\right] T_{1} Y_{12} & =0 .\end{cases}
$$

Note that conditions (23) and (24) guarantee a "strong PPT property". This is one of the most different aspects compared with definition in previous section. Similarly, we can define a subclass of SPPT states which satisfy condition (25) automatically.

Definition 5. Suppose that $\rho$ is an SPPT state with the decomposition of the form as Eq. (22). Then $\rho$ is called SSPPT if

$$
\left\{\begin{aligned}
{\left[S_{i}, S_{i}^{\dagger}\right] } & =0, i=1,2 \\
{\left[T_{i}, T_{i}^{\dagger}\right] } & =0, i=1,2 \\
{\left[S_{1}, T_{1}^{\dagger}\right] } & =0
\end{aligned}\right.
$$

In the following theorem, we show that SSPPT can guarantee the separability.

Theorem 2. All SSPPT states in the tripartite system $2 \otimes 2 \otimes N$ are separable.

Proof. According to the definition, $T_{1}, T_{2}, S_{1}, S_{2}$ are normal and $T_{1}$ commutes with $S_{1}$. Therefore, we have the following diagonalizations

$$
\begin{aligned}
& S_{1}=U \Sigma_{1} U^{\dagger}, T_{1}=U \Sigma_{2} U^{\dagger} \\
& S_{2}=V_{1} \Lambda_{1} V_{1}^{\dagger}, T_{2}=V_{2} \Lambda_{2} V_{2}^{\dagger}
\end{aligned}
$$

where $\Sigma_{i}, \Lambda_{i}$ are the diagonal matrices and $U, V_{1}$ and $V_{2}$ are all unitary matrices. Then 


$$
\begin{aligned}
X & =\left(\begin{array}{cccc}
U U^{\dagger} Y_{11} & U \Sigma_{2} U^{\dagger} Y_{11} & U \Sigma_{1} U^{\dagger} Y_{11} & U \Sigma_{1} \Sigma_{2} U^{\dagger} Y_{11} \\
0 & V_{1} V_{1}^{\dagger} Y_{12} & 0 & V_{1} \Lambda_{1} V_{1}^{\dagger} Y_{12} \\
0 & 0 & V_{2} V_{2}^{\dagger} Y_{21} & V_{2} \Lambda_{2} V_{2}^{\dagger} Y_{21} \\
0 & 0 & 0 & Y_{22}
\end{array}\right) \\
& =\left(\begin{array}{cccc}
U & 0 & 0 & 0 \\
0 & V_{2} & 0 & 0 \\
0 & 0 & V_{1} & 0 \\
0 & 0 & 0 & \mathbb{1}_{N}
\end{array}\right)\left(\begin{array}{cccc}
\tilde{Y}_{11} & \Sigma_{2} \tilde{Y}_{11} & \Sigma_{1} \tilde{Y}_{11} & \Sigma_{1} \Sigma_{2} \tilde{Y}_{11} \\
0 & \widetilde{Y}_{12} & 0 & \Lambda_{1} \tilde{Y}_{12} \\
0 & 0 & \tilde{Y}_{21} & \Lambda_{2} \tilde{Y}_{21} \\
0 & 0 & 0 & Y_{22}
\end{array}\right) \\
& =G \tilde{X}
\end{aligned}
$$

where

$$
\tilde{Y}_{11}=U^{\dagger} Y_{11}, \tilde{Y}_{12}=V_{1}^{\dagger} Y_{12}, \tilde{Y}_{21}=V_{2}^{\dagger} Y_{21}
$$

Let

$$
\begin{aligned}
& C_{1}=\left(\begin{array}{cccccc}
\tilde{Y}_{11} & \Sigma_{2} \tilde{Y}_{11} & \Sigma_{1} & \tilde{Y}_{11} & \Sigma_{1} \Sigma_{2} \tilde{Y}_{11}
\end{array}\right), \\
& C_{2}=\left(\begin{array}{cccc}
0 & \tilde{Y}_{12} & 0 & \Lambda_{1} \tilde{Y}_{12}
\end{array}\right), \\
& C_{3}=\left(\begin{array}{cccc}
0 & 0 & \tilde{Y}_{21} & \Lambda_{2} \tilde{Y}_{21}
\end{array}\right), \\
& C_{4}=\left(\begin{array}{cccc}
0 & 0 & 0 & Y_{22}
\end{array}\right) .
\end{aligned}
$$

Note that $\rho=X^{\dagger} X$ and $G$ is a unitary matrix, then we obtain,

$$
\rho=\sum_{i=1}^{4} C_{i}^{\dagger} C_{i} .
$$

On the other hand,

$$
\begin{aligned}
& C_{1}=\left(\mathbb{1}, \Sigma_{1}\right) \otimes\left(\mathbb{1}, \Sigma_{2}\right) \otimes \tilde{Y}_{11}, \\
& C_{2}=\left(\mathbb{1}, \Lambda_{1}\right) \otimes(0, \mathbb{1}) \otimes \tilde{Y}_{12}, \\
& C_{3}=(0, \mathbb{1}) \otimes\left(\mathbb{1}, \Lambda_{2}\right) \otimes \tilde{Y}_{21}, \\
& C_{4}=(0, \mathbb{1}) \otimes(0, \mathbb{1}) \otimes \tilde{Y}_{22} .
\end{aligned}
$$

It follows that each $C_{i}^{\dagger} C_{i}$ is separable, which implies the separability of $\rho$.

Note that this proof can also be served as a method to find the separability decomposition of a $2 \otimes 2 \otimes N$ SSPPT state.

Here we give a example of SPPT states with our definition.

Example 1. It was proved that every PPT state $\rho$ supported on $2 \otimes 2 \otimes N$ with rank $N$ is separable and has the canonical form [12]

$$
\rho=\sqrt{D}\left(\begin{array}{c}
\mathbb{1}_{N} \\
B^{\dagger} \\
C^{\dagger} \\
B^{\dagger} C^{\dagger}
\end{array}\right)\left(\begin{array}{llll}
\mathbb{1}_{N} & B & C & C B
\end{array}\right) \sqrt{D},
$$

where $B, C, D$ are operators in the third subsystem and $B, C$ are normal commuting matrices.

It is easy to check that this canonical form is SPPT in our definition. Forward, it is also an SSPPT state.

\section{B. SPPT states in $N_{1} \otimes N_{2} \otimes N_{3}$ tripartite system}

In this subsection we will extend the SPPT states to general tripartite system $N_{1} \otimes N_{2} \otimes N_{3}$. The basic idea is to require $\rho$ being SPPT under the bi-partition $A_{1}: A_{2} A_{3}$ and $A_{1} A_{2}: A_{3}$ simultaneously.

Let $\rho$ be the density matrix with a decomposition $\rho=$ $X^{\dagger} X$ in the tripartite system $N_{1} \otimes N_{2} \otimes N_{3}$.

Under the bipartite partition $A_{1}: A_{2} A_{3}, X$ can be written as an $N_{1} \times N_{1}$ block matrix,

$$
X=\left(\begin{array}{cccc}
X_{1} & S_{12} X_{1} & \cdots & S_{1 N_{1}} X_{1} \\
0 & X_{2} & \cdots & S_{2 N_{1}} X_{2} \\
\vdots & \vdots & \ddots & \vdots \\
0 & 0 & \cdots & X_{N_{1}}
\end{array}\right)=\left(\begin{array}{l}
S_{i j} X_{i} \\
\end{array}\right)
$$

where

$$
S_{i j}= \begin{cases}0, & \text { if } i>j, \\ \mathbb{1}_{N_{2} N_{3},}, & \text { if } i=j .\end{cases}
$$

Similarly, $X_{i}$ can be written as an $N_{2} \times N_{2}$ block matrix,

$$
\begin{aligned}
& X_{i}=\left(\begin{array}{cccc}
X_{i 1} & S_{i, 12}^{2} X_{i 1} & \cdots & S_{i, 1 N_{2}}^{2} X_{i 1} \\
0 & X_{i 2} & \cdots & S_{i, 2 N_{2}}^{2} X_{i 2} \\
\vdots & \vdots & \ddots & \vdots \\
0 & 0 & \cdots & X_{i N_{2}}
\end{array}\right) \\
& =\left(S_{i, k l}^{2} X_{i k}\right) \text {. }
\end{aligned}
$$

Here the superscript 2 in the matrices $S_{i, k l}^{2}$ indicates the subsystem $A_{2}$ and

$$
S_{i, k l}^{2}= \begin{cases}\mathbb{1}_{N_{3}}, & \text { if } k=l \\ 0, & \text { if } k>l .\end{cases}
$$


In order to be compatible with the SPPT structure in the bipartite system $A_{1} A_{2}: A_{3}$, we require $S_{i j}$ being diagonal,

$$
S_{i j}=\left(\begin{array}{cccc}
S_{i j, 1}^{1} & 0 & \cdots & 0 \\
0 & S_{i j, 2}^{1} & \cdots & 0 \\
\vdots & \vdots & \ddots & \vdots \\
0 & 0 & \cdots & S_{i j, N_{2}}^{1}
\end{array}\right)
$$

Hence

$$
\begin{aligned}
\rho & =X^{\dagger} X \\
X & =\left(S_{i j} X_{i}\right), \\
S_{i j} X_{i} & =\left(S_{i j, k}^{1} S_{i, k l}^{2} X_{i k}\right) .
\end{aligned}
$$

Now we are ready to define the SPPT state in general tripartite system with the matrices introduced above.

Definition 6. Let $\rho$ be the density matrix in the tripartite system $A_{1}: A_{2}: A_{3}$. It has a decomposition of the form as Eq. (34). Then we call $\rho$ SPPT w.r.t. the tripartite $A_{1}: A_{2}: A_{3}$ system (or simply SPPT), if $\rho$ is SPPT under the bi-partitions $A_{1}: A_{2} A_{2}$ and $A_{1} A_{2}: A_{3}$ simultaneously.

Note that the conditions for SPPT are equivalent to the following explicit matrix equations

$$
\begin{array}{r}
\sum_{i=1}^{N_{1}} X_{i}^{\dagger}\left[S_{i p}, S_{i q}^{\dagger}\right] X_{i}=0 \\
\sum_{i}^{N_{1}} \sum_{k}^{N_{2}} X_{i k}^{\dagger}\left[S_{i j, k}^{1} S_{i, k l}^{2},\left(S_{i j^{\prime}, k}^{1} S_{i, k l^{\prime}}^{2}\right)^{\dagger}\right] X_{i k}=0 .
\end{array}
$$

It is clear from the definition that SPPT states defined here are indeed PPT, i.e. positive under any partial transpose. As in previous subsection, we can also define a subclass of SPPT states with Eq. (35) satisfied.

Definition 7. Let $\rho$ be a state in tripartite system $N_{1} \otimes$ $N_{2} \otimes N_{3}$ with a decomposition $\rho=X^{\dagger} X$ of the form as Eq. (34). Then $\rho$ is said to be SSPPT if

$$
\begin{aligned}
{\left[S_{i p}, S_{i q}^{\dagger}\right] } & =0, \\
{\left[S_{i j, k}^{1} S_{i, k l}^{2},\left(S_{i j^{\prime}, k}^{1} S_{i, k l^{\prime}}^{\prime}\right)^{\dagger}\right] } & =0 .
\end{aligned}
$$

As in the bipartite system, we can prove SSPPT states are separable, which is a good property we want to keep.

Theorem 3. Every SSPPT state in tripartite system is separable.
Proof. Let $\rho$ be an SPPT in the tripartite system $N_{1} \otimes N_{2}$ $\otimes N_{3}$, which possesses a decomposition as Eq. (34). It follows from the condition (36) that $S_{i j}$ and $S_{i j^{\prime}}$ are commutable for any given $i$.

In particular, given $i, k$,

$$
S_{i j, k}^{1} \stackrel{\text { commutes }}{\longrightarrow} S_{i j^{\prime}, k}^{1} \text {. }
$$

Note that if we put $l=k$ and $j^{\prime}=i$ in Eq. (36), then for any given $i, k$ we have

$$
S_{i j, k}^{1} \stackrel{\text { commutes }}{\longrightarrow} S_{i, k l^{\prime}}^{2} .
$$

In the similar way, let $j=i$ and $j^{\prime}=i$ in Eq. (36), we obtain,

$$
S_{i, k l^{\prime}}^{2} \stackrel{\text { commutes }}{\longrightarrow} S_{i, k l}^{2} \text {. }
$$

Therefore we have a simultaneous diagonalizations,

$$
\begin{aligned}
& S_{i j, k}^{1}=U_{i k} \Lambda_{i j k}^{1} U_{i k}^{\dagger}, \\
& S_{i, k l}^{2}=U_{i k} \Lambda_{i k l}^{2} U_{i k}^{\dagger},
\end{aligned}
$$

where $U_{i k}$ are unitary matrices and $\Lambda_{i j k}^{1}, \Lambda_{i k l}^{2}$ are diagonal matrices with

$$
\begin{aligned}
\Lambda_{i j k}^{1} & =\operatorname{diag}\left(\lambda_{i j k 1}^{1}, \lambda_{i j k 2}^{1}, \ldots, \lambda_{i j k N_{3}}^{1}\right), \\
\Lambda_{i k l}^{2} & =\operatorname{diag}\left(\lambda_{i k l 1}^{2}, \lambda_{i k l 2}^{2}, \ldots, \lambda_{i k l N_{3}}^{2}\right) .
\end{aligned}
$$

Let

$$
\begin{aligned}
U & =\left(\begin{array}{cccc}
U_{1} & 0 & \cdots & 0 \\
0 & U_{2} & \cdots & 0 \\
\vdots & \vdots & \ddots & \vdots \\
0 & 0 & \cdots & U_{N_{1}}
\end{array}\right) \\
\tilde{X} & =\left(\begin{array}{cccc}
Y_{11} & Y_{12} & \cdots & Y_{1 N_{1}} \\
Y_{21} & Y_{22} & \cdots & Y_{2 N_{1}} \\
\vdots & \vdots & \ddots & \vdots \\
Y_{N_{1}, 1} & Y_{N_{1}, 2} & \cdots & Y_{N_{1}, N_{1}}
\end{array}\right),
\end{aligned}
$$

where

$$
\begin{aligned}
U_{i} & =\left(\begin{array}{cccc}
U_{i 1}^{\dagger} & 0 & \cdots & 0 \\
0 & U_{i 2}^{\dagger} & \cdots & 0 \\
\vdots & \vdots & \ddots & \vdots \\
0 & 0 & \cdots & U_{i, N_{2}}^{\dagger}
\end{array}\right), \\
Y_{i j} & =\left(\begin{array}{ccc}
\Lambda_{i j k}^{1} \Lambda_{i k l}^{2} X_{i k}
\end{array}\right) \\
\widetilde{X}_{i k} & =U_{i k}^{\dagger} X_{i k} .
\end{aligned}
$$

Since $U$ is unitary and $X=U \widetilde{X}$, we have $\rho=\tilde{X}^{\dagger} \tilde{X}$.

Suppose $\tilde{X}_{i k}=\left(a_{i k 1}, a_{i k 2}, \ldots, a_{i k N_{3}}\right)^{\top}$ where each $a_{i k l}$ is a row vectors in $\mathbb{C}^{N_{3}}$ space. Now consider the 
$n(i, k, p)$-th row of $\tilde{X}$, which is denoted by $v_{i k p}$. Then we have

$$
v_{i k p}=w_{i k p} \otimes a_{i k p}
$$

where

$$
\begin{aligned}
w_{i k p} & =\left(y_{i k p 1}, y_{i k p 2}, \ldots, y_{i k p N_{2}}\right) \\
y_{i k p j} & =\left(\lambda_{i j k p}^{1} \lambda_{i k 1 p}^{2}, \lambda_{i j k p}^{1} \lambda_{i k 2 p}^{2}, \ldots, \lambda_{i j k p}^{1} \lambda_{i k N_{2} p}^{2}\right) \\
& =\lambda_{i j k p}^{1}\left(\lambda_{i k 1 p}^{2}, \lambda_{i k 2 p}^{2}, \ldots, \lambda_{i k N_{2} p}^{2}\right) .
\end{aligned}
$$

It follows that each $v_{i k p}$ is a product vector,

$$
\begin{aligned}
v_{i k p}= & \left(\lambda_{i 1 k p}^{1}, \ldots, \lambda_{i N_{1} k p}^{1}\right) \\
& \otimes\left(\lambda_{i k 1 p}^{2}, \ldots, \lambda_{i k N_{2} p}^{2}\right) \otimes a_{i k p} .
\end{aligned}
$$

Therefore $\rho$ is separable.

This proof can also be utilized as a method to find the separability decomposition of SSPPT states in tripartite system. Now we end this subsection by given some examples of tripartite SPPT states.

Example 2. Recall that a state $\rho$ on $N_{1} \otimes N_{2}$ is said to be a CQ state [21] if it has the form

$$
\rho=\sum_{i}^{N_{1}} p_{i}|i\rangle\langle i| \otimes \rho_{i}^{A_{2}}
$$

Where $\rho_{i}^{A_{2}}$ are density matrices in $A_{2}$ subsystem. It was proved that any $C Q$ state is in fact SSPPT state. Similarly, we construct a class of SPPT states in tripartite system $A_{1}: A_{2}: A_{3}$ as follows,

$$
\rho=\sum_{i}^{N_{1}} \sum_{j}^{N_{2}} p_{i j}|i j\rangle\langle i j| \otimes \rho_{i j}^{A_{3}},
$$

where $\rho_{i j}^{A_{3}}$ are density matrices in subsystem $A_{3}$. This is in fact an SSPPT states with $S_{i j, k}^{1}=\delta_{i j} I, S_{i, k l}^{2}=\delta_{k l} I$ and $X_{i k}=p_{i k} \rho_{i k}^{A_{3}}$.

Example 3. Recall that every PPT state $\rho$ in $N_{1} \otimes N_{2}$ $\otimes N_{3}$ with $\operatorname{rank}(\langle 00|\rho| 00\rangle)=\operatorname{rank}(\rho)=N_{3}$ can be transformed into the following canonical form by using a reversible local operator [22],

$$
\rho=T^{\dagger} T
$$

where

$$
T=\left(\mathbb{1}_{N_{3}}, A_{2}, \ldots, A_{N_{1}}\right) \otimes\left(\mathbb{1}_{N_{3}}, B_{2}, \ldots, B_{N_{2}}\right) .
$$

Moreover $A_{i}, B_{i}$ are a set of normal commuting matrices.

Now we show this canonical form is actually an SSPPT state.

Assume $A_{1}=B_{1}=\mathbb{1}_{N_{3}}$ and $X_{i j}=\mathbb{1}_{N_{3}}, \forall i, j$. Let $S_{1, j, 1}^{1}=A_{j}$ and $S_{1,1 l}^{2}=B_{l}$ and all the other $S_{i j, k}^{1}, S_{i, k l}^{2}$ are zero matrices. Then $T$ coincides with $X$ in Eq. (34). Since all the $S_{i j, k}^{1}$ and $S_{i, k l}^{2}$ are normal commuting, $\rho$ is SSPPT.

\section{SPPT states in multipartite system}

In this subsection, we will finally give the definition of SPPT in $(d+1)$-particle system $N_{1} \otimes N_{2} \otimes \cdots \otimes N_{d} \otimes N_{0}$. To begin with, we will fix some notations for representing matrices in the multipartite system.

Let $\alpha_{n}=\left(i_{1}, i_{2}, \ldots, i_{n}\right)$ with $i_{k} \in\left\{1,2, \ldots, N_{k}\right\}, k$ $\in\{1,2, \ldots, d\}$. Similarly, let $\beta_{n}=\left(j_{1}, j_{2}, \ldots, j_{n}\right)$. Note that the indexes $i_{n}, j_{n}$ correspond to the $n$-th subsystem. For simplicity, we write $\alpha_{d}, \beta_{d}$ as $\alpha, \beta$. Hence we can represent the matrices in a conciser form. For example,

$$
\begin{aligned}
X_{\alpha_{n}} & =X_{i_{1}, i_{2}, \ldots, i_{n}}, \\
S_{\alpha_{n}, j_{m}} & =S_{i_{1}, i_{2}, \ldots, i_{m}, j_{m}, i_{m+1}, \ldots, i_{n}}, m \leqslant n .
\end{aligned}
$$

Hereafter in this subsection, $(\alpha, \beta)$-th entry of a matrix to represent the element in $n(\alpha)$-th row and $n(\beta)$-th column.

Let $\rho=X^{\dagger} X$ be a density matrix in the $N_{1} \otimes N_{2} \otimes \cdots$ $\otimes N_{d} \otimes N_{0}$ system. Consider the following class of upper triangular block matrix $X$, whose elements are $N_{0} \times N_{0}$ matrices. The $(\alpha, \beta)$-th entry of $X$ is

$$
\prod_{p=1}^{d} S_{\alpha, j_{p}}^{p} X_{\alpha}, \quad S_{\alpha, j_{p}}^{p}, X_{\alpha} \in \mathbb{C}^{N_{0} \times N_{0}},
$$

where

$$
\begin{aligned}
& S_{\alpha_{n}, j_{p}}^{p}=\operatorname{diag}\left(S_{\alpha_{n}, 1, j_{p}}^{p}, \ldots, S_{\alpha_{n}, N_{n+1}, j_{p}}^{p}\right), \\
& X_{\alpha_{n}}=\left(S_{\alpha_{n}, i_{n+1}, j_{n+1}}^{n+1} X_{\alpha_{n}, i_{n+1}}\right) \\
& =\left(\begin{array}{ll}
S_{\alpha_{n+1}, j_{n+1}}^{n+1} & X_{\alpha_{n+1}}
\end{array}\right), \\
& S_{\alpha_{n}, j_{p}}^{p}= \begin{cases}1, & j_{p}=i_{p} \\
0, & j_{p}<i_{p}\end{cases}
\end{aligned}
$$

Definition 8. Let $\rho$ be the density matrix in the $(d+1)$ body system $N_{1} \otimes \cdots \otimes N_{d} \otimes N_{0}$ with the decomposition $\rho=X^{\dagger} X$ of the form as Eq. (49). Then $\rho$ is said to be SPPT if

$$
\sum_{\alpha_{n}} X_{\alpha_{n}}^{\dagger}\left[\prod_{p=1}^{n} S_{\alpha_{n}, j_{p}}^{p},\left(\prod_{q=1}^{n} S_{\alpha_{n}, j_{q}^{\prime}}^{q}\right)^{\dagger}\right] X_{\alpha_{n}}=0
$$

for any $\beta_{n}=\left(j_{1}, j_{2}, \ldots, j_{n}\right), \beta_{n}^{\prime}=\left(j_{1}^{\prime}, j_{2}^{\prime}, \ldots, j_{n}^{\prime}\right)$ and $n=1,2, \ldots, d$.

The following theorem shows that this generalization of SPPT preserves the PPT property.

Theorem 4. Any SPPT state is PPT.

Proof. Consider the density matrix $\rho$ in the $N_{1} \otimes \cdots N_{d} \otimes$ $N_{0}$ system. Suppose $\rho=X^{\dagger} X$, where $X$ has the form 
as Eq. (49). Recall a special partial transpose defined previously,

$$
\Gamma_{n}=\mathrm{T}_{\{1,2, \ldots, n\}}, n=1,2, \ldots, d .
$$

To prove the PPT property of $\rho$, it suffice to show that $\rho^{\Gamma_{n}}$ is positive for any $n$.

Consider the state under the bi-partition $A_{1} A_{2} \ldots A_{n}$ : $A_{n+1} \ldots A_{d} A_{0}$. Then $X$ can be regarded as a $r \times r$ block matrix, where $r=\prod_{k=1}^{n} N_{k}$. Given any $\alpha_{n}=$ $\left(i_{1}, i_{2}, \ldots, i_{n}\right)$ and $\beta_{n}=\left(j_{1}, j_{2}, \ldots, j_{n}\right)$, the $\left(\alpha_{n}, \beta_{n}\right)$-th entry of $X$ is

$$
\prod_{p=1}^{n} S_{\alpha_{n}, j_{p}}^{p} X_{\alpha_{n}}
$$

Let $Y$ be the matrix whose $\left(\alpha_{n}, \beta_{n}\right)$-th entry is

$$
\left(\prod_{p=1}^{n} S_{\alpha_{n}, j_{p}}^{p}\right)^{\dagger} X_{\alpha_{n}}
$$

According to the conditions (50), we have $\rho^{\Gamma_{n}}=Y^{\dagger} Y$, which completes the proof.

In a similar way to tripartite system, we can define a special sub-class of SPPT states.

Definition 9. Let $\rho=X^{\dagger} X$ be an SPPT state where $X$ has the form as Eq. (49). Then $\rho$ is said to be SSPPT if

$$
\left[\prod_{p=1}^{n} S_{\alpha_{n}, j_{p}}^{p},\left(\prod_{q=1}^{n} S_{\alpha_{n}, j_{q}^{\prime}}^{q}\right)^{\dagger}\right]=0
$$

for any $\alpha_{n}, \beta_{n}, \beta_{n}^{\prime}$ and $n=1,2, \ldots, d$.

The following theorem shows that SSPPT guarantees the separability in an arbitrary multipartite system.

Theorem 5. Any SSPPT state is separable.

Proof. Let $\rho$ be the density matrix in the $(d+1)$-particle system $N_{1} \otimes \cdots N_{d} \otimes N_{0}$. Suppose $\rho=X^{\dagger} X$ with $X$ being of the form as Eq. (49).

Consider the condition (51). Given $k, l$, choose $\alpha_{n}$ and $\beta_{n}$ such that

$$
\begin{aligned}
& j_{p}=i_{p}, p=1, \ldots, n, p \neq k \\
& i_{q^{\prime}}=i_{q}, q=1, \ldots, n, q \neq l .
\end{aligned}
$$

It follows that

$$
S_{\alpha_{n}, j_{p}}^{p}=S_{\alpha_{n}, j_{q}^{\prime}}^{q}=\mathbb{1}, p \neq k, q \neq l,
$$

which implies that

$$
\left[S_{\alpha_{n}, j_{k}}^{k} S_{\alpha_{n}, j_{l}^{\prime}}^{l}\right]=0
$$

Forward by the structure of $S_{\alpha_{n}, j_{p}}^{p}$, we have

$$
\left[S_{\alpha, j_{k}}^{k} S_{\alpha, j_{l}^{\prime}}^{l}\right]=0
$$

That is to say $\left\{S_{\alpha, j_{p}}^{p}\right\}$ is a set of normal commuting matrices for fixed $\alpha$.

Hence we have the simultaneous diagonalizations

$$
S_{\alpha, j_{p}}^{p}=U_{\alpha} \Lambda_{\alpha, j_{p}}^{p} U_{\alpha}^{\dagger}
$$

where $U_{\alpha}$ is an $N_{0} \times N_{0}$ unitary matrix and $\Lambda_{\alpha, j_{p}}^{p}$ are diagonal matrices with

$$
\Lambda_{\alpha, j_{p}}^{p}=\operatorname{diag}\left(\lambda_{\alpha, 1, j_{p}}^{p}, \lambda_{\alpha, 2, j_{p}}^{p}, \ldots, \lambda_{\alpha, N_{0}, j_{p}}^{p}\right) .
$$

Let $U$ be the block matrix whose $(\alpha, \alpha)$-th entry is $U_{\alpha}$ and other entries are zero. Put $\tilde{X}=U X$ and $\tilde{X}_{\alpha}=U_{\alpha} X_{\alpha}$, then $\rho=\tilde{X}^{\dagger} \tilde{X}$.

Let

$$
\tilde{X}_{\alpha}=\left(\begin{array}{c}
a_{\alpha, 1} \\
a_{\alpha, 2} \\
\vdots \\
a_{\alpha, N_{0}}
\end{array}\right) \text {, }
$$

where each $a_{\alpha, i_{0}}$ is a row vector in $\mathbb{C}^{N_{0}}$. Note that $(\alpha, \beta)$ th entry of $\tilde{X}$ is

$$
\left(\prod_{p=1}^{n} \Lambda_{\alpha, j_{p}}\right) \tilde{X}_{\alpha}
$$

Hence we have,

$$
v_{\alpha, i_{0}}=w_{\alpha, i_{0}} \otimes a_{\alpha, i_{0}}
$$

where

$$
\begin{aligned}
w_{\alpha, i_{0}} & =\otimes_{p=1}^{d} y_{\alpha, i_{0}}^{p}, \\
y_{\alpha, i_{0}}^{p} & =\left(\lambda_{\alpha, i_{0}, 1}^{p}, \lambda_{\alpha, i_{0}, 2}^{p}, \ldots, \lambda_{\alpha, i_{0}, N_{p}}^{p}\right) \in \mathbb{C}^{N_{p}} .
\end{aligned}
$$

Now that each row of $\tilde{X}$ is a product vector, it follows that $\rho$ is separable.

The following lemma shows an example of SPPT state in the general multipartite system.

Lemma 6. Any pure state is separable if and only if it is SPPT.

Proof. Since any pure PPT state is separable, it suffices to prove that pure product state is indeed SPPT states. Let $\rho$ be a pure state in $N_{1} \otimes \cdots N_{d} \otimes N_{0}$ system. Hence $\rho$ can be written as

$$
\begin{aligned}
\rho & =v v^{\dagger}, v=w^{\dagger}, \\
w & =\left(\otimes_{i=1}^{d} w_{i}\right) \otimes w_{0}, \\
w_{p} & =\left(w_{p, 1}, w_{p, 2}, \ldots, w_{p, N_{p}}\right), 1 \leqslant p \leqslant d .
\end{aligned}
$$

Let $\alpha_{1}=(1,1, \ldots, 1)$ and $X_{\alpha_{1}}$ be a $N_{0} \times N_{0}$ matrix whose first row is $w_{0}$ and all other entries are zeros. Consider $w$ as a block vector with each block being a $N_{0}$ dimensional row vector, then the $\alpha$-entry of $w$ is $\prod_{p}^{d} w_{p, j_{p}} w_{0}$. 
Let $\beta=\left(j_{1}, j_{2}, \ldots, j_{d}\right)$ and

$$
S_{\alpha_{1}, j_{p}}^{p}=\operatorname{diag}\left(w_{p, j_{p}}, 0, \ldots, 0\right) .
$$

For any other $\alpha \neq \alpha_{1}$, let $S_{\alpha, j_{p}}^{p}=0$ and $X_{\alpha}=0$. Then we can write $\rho$ as $\rho=X^{\dagger} X$, where the $(\alpha, \beta)$-th entry of $X$ is

$$
\prod_{p=1}^{d} S_{\alpha, j_{p}}^{p} X_{\alpha}
$$

which has the same structure as in the definition of SPPT. Moreover, $S_{\alpha, p}^{p}$ here are all commuting normal matrices, hence it is SSPPT.

We end this subsection by giving another example in the multipartite system.

Example 4. It was proved that any PPT state in $N_{1} \otimes \cdots$ $\otimes N_{d} \otimes N_{0}$ is separable [22] if

$$
\begin{aligned}
\operatorname{rank}(\rho) & =\operatorname{rank}\left(\left\langle 0_{1}, 0_{2}, \ldots, 0_{d}|\rho| 0_{1}, 0_{2}, \ldots, 0_{d}\right\rangle\right) \\
& =N_{0} .
\end{aligned}
$$

It then has a canonical form by using a reversal local operator:

$$
\rho=T^{\dagger} T
$$

where

$$
\begin{aligned}
T= & \left(D_{1}^{1}, D_{2}^{1}, \ldots, D_{N_{1}}^{1}\right) \otimes\left(D_{1}^{2}, D_{2}^{2}, \ldots, D_{N_{1}}^{2}\right) \cdots \\
& \otimes\left(D_{1}^{d}, D_{2}^{d}, \ldots, D_{N_{d}}^{d}\right) .
\end{aligned}
$$

Here $D_{1}^{i}=\mathbb{1}$ and $D_{p}^{q}$ are a set of mutually commuting normal matrices.

Suppose that $X$ has the form as Eq. (49). Let $\alpha_{0}=$ $(1,1, \ldots, 1)$ be a $d$-tuple. Put $S_{\alpha_{0}, j_{p}}^{p}=D_{j_{p}}^{p}$ for any $p$. And $S_{\alpha, j_{p}}^{p}=0$ for all other $\alpha \neq \alpha_{0}$. And $X_{\alpha_{0}}=\mathbb{1}$. Simple calculation follows that $\rho=X^{\dagger} X$. Note that $S_{\alpha, j_{p}}^{p}$ are all mutually normal commuting, hence it is SSPPT.

\section{SUFFICIENT SEPARABILITY CONDITIONS OF SPPT STATES}

In this section, we will consider the separability conditions for SPPT state.

Let $\rho$ be a density matrix in $2 \otimes d$ system with a block Cholesky decomposition,

$$
\begin{aligned}
\rho & =X^{\dagger} X, \\
X & =\left(\begin{array}{cc}
X_{1} & S X_{1} \\
0 & X_{2}
\end{array}\right) .
\end{aligned}
$$

Note that $\rho$ is SPPT if

$$
X_{1}^{\dagger}\left(S_{1} S_{1}^{\dagger}-S_{1}^{\dagger} S_{1}\right) X_{1}=0 .
$$

It has been proved that SPPT states in $2 \otimes 4$ system is separable [24]. In fact we have the following conclusion.
Lemma 7. Let $\rho$ be an SPPT state of the form as Eq. (61). Then $\rho$ is separable in either of the following cases
1. $d \leqslant 4$;
2. $\operatorname{rank}\left(X_{1}\right)=d$.

The second condition can be further improved as follows.

Lemma 8. Let $\rho$ be an SPPT state of the form as Eq. (61). Then $\rho$ is separable if $\operatorname{Im}(S) \subset \operatorname{Im}\left(X_{1}\right)$ or $\operatorname{Im}\left(S^{\dagger}\right) \subset \operatorname{Im}\left(X_{1}\right)$.

Proof. It suffices to prove for the case $\operatorname{Im}(S) \subset \operatorname{Im}\left(X_{1}\right)$ since otherwise we can consider the partial transposed state $\rho^{\mathrm{T}_{1}}$.

Suppose $\operatorname{rank}(X)<d$, then it has a SVD decomposition,

$$
X_{1}=U \Lambda V^{\dagger}=U\left(\begin{array}{cc}
\Sigma & 0 \\
0 & 0
\end{array}\right) V^{\dagger}
$$

where $U, V$ are unitary matrices and $\Sigma$ is a diagonal matrix with dimension less than $d$. Let

$$
\begin{aligned}
\sigma & =Y^{\dagger} Y, \\
Y & =\left(\begin{array}{cc}
\Gamma & U^{\dagger} S U \Gamma \\
0 & X_{2} V
\end{array}\right) .
\end{aligned}
$$

Then $\rho=(\mathbb{1} \otimes V) \sigma\left(\mathbb{1} \otimes V^{\dagger}\right)$. Simple calculation gives that $\sigma$ is also SPPT. Write $S$ in block matrix form according to that of $\Lambda$,

$$
S=\left(\begin{array}{ll}
S_{1} & S_{2} \\
S_{3} & S_{4}
\end{array}\right)
$$

Note that

$$
\operatorname{Im}(S) \subset \operatorname{Im}\left(X_{1}\right) \Leftrightarrow \operatorname{Im}\left(U^{\dagger} S U\right) \subset \operatorname{Im}(\Lambda),
$$

it follows that $S_{3}=0, S_{4}=0$. By the condition (62), we have $S_{4}=0$ and $S_{1}$ is normal. Therefore $S$ is normal, which implies $\rho$ is separable.

Another sufficient condition for separability of $2 \otimes d$ SPPT state was given in Ref. [19].

Lemma 9. Let

$$
\rho=\left(\begin{array}{cc}
A & B \\
B^{\dagger} & D
\end{array}\right)
$$

be a density matrix in the $2 \otimes d$ system. If $A>D$, then $\rho$ is SSPPT and thus separable.

Noted that, when $\rho$ is written in Eq. (61), the sufficient condition in the above lemma is equivalent to

$$
X_{1}^{\dagger} X_{1}>X_{1}^{\dagger} S^{\dagger} S X_{1}+X_{2}^{\dagger} X_{2} .
$$

We can further relax the condition by 
Lemma 10. Let $\rho=X^{\dagger} X$ with $X$ being of the form as $E q$. (61) in $2 \otimes d$ system. Then $\rho$ is separable if

$$
X_{1}^{\dagger} X_{1}>X_{1}^{\dagger} S^{\dagger} S X_{1} .
$$

Proof. Now $\rho$ can be written as

$$
\begin{aligned}
\rho= & \left(\begin{array}{cc}
X_{1}^{\dagger} S^{\dagger} S X_{1} & X_{1}^{\dagger} S X_{1} \\
X_{1}^{\dagger} S^{\dagger} X_{1} & X_{1}^{\dagger} S^{\dagger} S X_{1}
\end{array}\right) \\
& +\left(\begin{array}{cc}
X_{1} X_{1}^{\dagger}-X_{1}^{\dagger} S^{\dagger} S X_{1} & 0 \\
0 & X_{2}^{\dagger} X_{2}
\end{array}\right) .
\end{aligned}
$$

The former term is a positive Toeplitz block matrix which is separable by the Proposition 1 in Ref. [23]. Since the latter term is separable, $\rho$ is separable.

To sum up the conditions in term of $S$, we have

Corollary 11. Let $\rho$ be an SPPT state of the form as $E q$. (61). Then $\rho$ is separable if $S$ is in any of the following casesa

1. $S$ is contractive,

2. $S$ is normal,

3. $\operatorname{Im}(S) \subset \operatorname{Im}\left(X_{1}\right)$,

4. Dimension of $S$ is less than or equal to 4.

Kil-Chan Ha constructed a $2 \otimes 5$ SPPT state which is entangled [16]. Here we study further about $2 \otimes 5 \mathrm{SPPT}$ states. Before that we recall the definition of edge state.

Definition 10. Let $\sigma$ be multipartite state. It is said to be an edge state if there does not exist $|x, y\rangle$ such that

$$
\begin{aligned}
|x, y\rangle & \in \mathcal{R}(\sigma), \\
\left|x^{*}, y\right\rangle & \in \mathcal{R}\left(\sigma^{T_{1}}\right) .
\end{aligned}
$$

Theorem 12. $\rho$ is an SPPT state in $2 \otimes 5$ system of the form as Eq. (61), then $\rho$ is separable except the following case:

$$
\left\{\begin{array}{l}
\operatorname{rank}\left(X_{1}\right)=4 \\
\operatorname{rank}(\sigma)=\operatorname{rank}\left(\sigma^{T_{1}}\right)=5 \\
\sigma \text { is an edge state }
\end{array}\right.
$$

where

$$
\sigma=W^{\dagger} W, \quad W=\left(\begin{array}{ll}
X_{1} & S X_{1}
\end{array}\right) .
$$

Proof. By Lemma 7, $\rho$ is separable if it has full rank. Hence we assume $r=\operatorname{rank}\left(X_{1}\right) \leqslant 4$. Consider $\sigma$, an SPPT state supported in $2 \otimes r$ subspace. If $r<4$, then it is separable by the Peres-Horodecki criterion. Therefore, $\rho$ is separable. We are thus able to assume $r=4$.

By the PPT property, $\operatorname{rank}(\sigma) \geqslant r=4$. And it is separable if $\operatorname{rank}(\sigma)=4 \operatorname{or} \operatorname{rank}\left(\sigma^{\mathrm{T}_{1}}\right)=4$. Hence we only need to consider the case when $\operatorname{rank}(\sigma)=\operatorname{rank}\left(\sigma^{T_{1}}\right)=5$.

Since any $2 \otimes 4$ birank $(5,5)$ state is entangled if and only if it is an edge state. Hence $\rho$ is separable when $\sigma$ is not an edge state, which completes our proof.
Recall the $2 \otimes 5$ SPPT entangled state in Ha's paper [24],

$$
X_{1}=\left(\begin{array}{cc}
\mathbb{1}_{4} & 0 \\
0 & 0
\end{array}\right), S=\left(\begin{array}{ccccc}
0 & 1 & 0 & 0 & \beta_{1} \\
0 & 0 & 1 & 0 & 0 \\
0 & 0 & 0 & 1 & 0 \\
0 & 0 & 0 & 0 & \beta_{2} \\
\beta_{2} & 0 & 0 & \beta_{1} & 0
\end{array}\right)
$$

where $\beta_{1}=\sqrt{(1-b) / 2 b}$ and $\beta_{2}=\sqrt{(1+b) / 2 b}$ with $0<b<1$. Put $X_{2}=0$. Then the defined $\sigma$ is

$$
\sigma=\left(\begin{array}{ccccc|ccccc}
1 & 0 & 0 & 0 & 0 & 0 & 1 & 0 & 0 & 0 \\
0 & 1 & 0 & 0 & 0 & 0 & 0 & 1 & 0 & 0 \\
0 & 0 & 1 & 0 & 0 & 0 & 0 & 0 & 1 & 0 \\
0 & 0 & 0 & 1 & 0 & 0 & 0 & 0 & 0 & 0 \\
0 & 0 & 0 & 0 & 0 & 0 & 0 & 0 & 0 & 0 \\
0 & 0 & 0 & 0 & 0 & \gamma_{1} & 0 & 0 & \gamma_{2} & 0 \\
1 & 0 & 0 & 0 & 0 & 0 & 1 & 0 & 0 & 0 \\
0 & 1 & 0 & 0 & 0 & 0 & 0 & 1 & 0 & 0 \\
0 & 0 & 1 & 0 & 0 & \gamma_{2} & 0 & 0 & \gamma_{1} & 0 \\
0 & 0 & 0 & 0 & 0 & 0 & 0 & 0 & 0 & 0
\end{array}\right),
$$

where $\gamma_{1}=(b+1) / 2 b, \gamma_{2}=\sqrt{b^{2}-1} . \sigma$ is supported on $2 \otimes 4$ subspace and is of birank $(5,5)$ state. By computing all the product vectors in range $\sigma$ and $\sigma^{T_{1}}$ respectively, it follows that $\sigma$ is an edge state, which coincides with our theorem.

Furthermore, we have studied the rank 4 SPPT state.

Theorem 13. Any SPPT state of rank less than or equal to 4 is separable.

Proof. Since all the rank 1,2, and 3 PPT states are separable, it only remains to consider the rank 4 states. It has been proved in Refs. [25, 26] that any rank four PPT state is separable except in the $2 \otimes 2 \otimes 2$ and $3 \otimes 3$ systems. And for these systems, the state is separable if and only if its range contains a product vector. Therefore, it suffices to prove that $\mathcal{R}(\rho)$ contains a product vector in $2 \otimes 2 \otimes 2$ and $3 \otimes 3$ systems respectively.

Firstly, we consider the $2 \otimes 2 \otimes 2$ case. Let $\rho=X^{\dagger} X$ be an SPPT state in $2 \otimes 2 \otimes 2$ system, where $X$ satisfies conditions (22) and (25). Note that $\rho$ has a product vector in its range is equivalent to that $X$ has a product vector in its row range.

If $Y_{22} \neq 0$, then $\mathcal{R}\left(X^{\top}\right)$ contains a product vector.

Let $\rho^{\prime}=\left(Y_{21}, T_{2} Y_{21}\right)^{\dagger}\left(Y_{21}, T_{2} Y_{21}\right)$, then $\rho^{\prime}$ is a $2 \otimes 2$ state. It is known from Ref. [27] that any two dimensional subspace of $2 \otimes 2$ system always contains a product vector. If $\operatorname{rank}\left(Y_{21}, T_{2} Y_{21}\right)=2$, then $\rho^{\prime}$ contains a product vector in its range, namely $u$. Moreover, $(0,1) \otimes u$ is a product vector in the range of $\rho$. On the other hand, if $\operatorname{rank}\left(Y_{21}, T_{2} Y_{21}\right)=1$, we claim that $\rho$ also contains a product vector in its range. Consider the SVD decomposition of $Y_{21}$, denoted by $Y_{21}=U \Sigma V^{\dagger}$. Then the 5,6 -th rows of $X$ is

$$
U\left(\begin{array}{cccccccc}
0 & 0 & 0 & 0 & \sigma & 0 & t_{1} \sigma & 0 \\
0 & 0 & 0 & 0 & 0 & 0 & t_{2} \sigma & 0
\end{array}\right) V^{\dagger}
$$


where

$$
\Sigma=\left(\begin{array}{ll}
\sigma & 0 \\
0 & 0
\end{array}\right), U^{\dagger} T_{2} U=\left(\begin{array}{cc}
t_{1} & t_{3} \\
t_{2} & t_{4}
\end{array}\right)
$$

Note that $\left(0,0,0,0, \sigma, 0, t_{1} \sigma, 0\right)$ is always a product vector for any $t_{1}$.

Similar way, we can show that $\rho$ contains a product vector in its range if $Y_{12} \neq 0$.

However, $\operatorname{rank}(\rho)=4$ contradicts with $Y_{12}=0, Y_{21}=$ 0 , and $Y_{22}=0$. It follows than $\rho$ is separable.

Next, we consider the case when $\rho$ is in $3 \otimes 3$ bipartite system. Let $\rho$ be an SPPT state with

$$
\begin{aligned}
\rho & =X^{\dagger} X \\
X & =\left(\begin{array}{ccc}
X_{1} & S_{12} X_{1} & S_{13} X_{1} \\
0 & X_{2} & S_{23} X_{2} \\
0 & 0 & X_{3}
\end{array}\right) .
\end{aligned}
$$

If $X_{3} \neq 0$, then $\rho$ contains a product in its range.

Now assume $X_{3}=0$. If $X_{1}$ has full rank, then $\left(X_{2}, S_{23} X_{2}\right)$ must be rank one. Suppose

$$
X_{2}=\left(\begin{array}{c}
\lambda_{1} a \\
\lambda_{2} a \\
\lambda_{3} a
\end{array}\right)
$$

where $a$ is a row vector in $\mathbb{C}^{3}$. The row range of $S_{23} X_{2}$ will be contained in that of $X_{2}$, we have

$$
S_{23} X_{2}=\left(\begin{array}{c}
\sigma_{1} a \\
\sigma_{2} a \\
\sigma_{3} a
\end{array}\right)
$$

for some $\sigma_{i}$.

However $\left(0, \lambda_{i}, \sigma_{i}\right) \otimes a$ is a product vector in the row range of $\rho$.
Therefore, it only remains to consider the case when $\operatorname{rank}\left(X_{1}\right)=2$.

Since the rank of $\rho$ is 4 , then $1 \leqslant \operatorname{rank}\left(X_{2}\right) \leqslant 2$. It has been proved that $\operatorname{rank}\left(X_{2}\right)=1$ implies the separability of $\rho$, we need only to consider the case when $\operatorname{rank}\left(X_{2}\right)=2$.

Let $\sigma=\left(0, X_{2}, S_{23} X_{2}\right)^{\dagger}\left(0, X_{2}, S_{23} X_{2}\right)$ which is supported in $2 \otimes 2$ subspace. Since any two dimensional subspace in $2 \otimes 2$ system contains at least one product vector, $\rho$ must contain a product vector in its range.

Above all, we conclude that any rank 4 SPPT state is separable.

\section{CONCLUSION}

We extend the concept of well-known SPPT states to the arbitrary n-body system. We compare the difference between the definition of SPPT in Ref. [20] and ours. It turns out that our states can inherit the structure of PPT and many good properties as those in the bipartite system. For example, any SPPT states are separable, pure states are separable if and only if they are SPPT, and any SPPT state of rank 4 is separable. Besides, we also give some sufficient conditions for separability of SPPT states. In particular, for the $2 \otimes 5$ SPPT states, we showed that most of the states are separable except a special subclass. We hope our work will be helpful for investigating the structure of multipartite PPT states.

\section{ACKNOWLEDGMENTS}

The author is supported by an NUS Research Scholarship. The author would like to thank the support and supervision from Professor Chu Delin. The author also gratefully acknowledges Mr. Cui Hanwen for the checking of the final script. Finally, the author would specifically like to highlight the impetus behind this work by Ms. Liu Cuizhen.
[1] A. Einstein, B. Podolsky, and N. Rosen, Phys. Rev. 47, 777 (1935).

[2] R. F. Werner, Phys. Rev. A 40, 4277 (1989).

[3] A. Peres, Phys. Rev. Lett. 77, 1413 (1996).

[4] L. Gurvits, in Proceedings of the thirty-fifth ACM symposium ACM (ACM Press, 2003) pp. 10-19.

[5] M. Horodecki, P. Horodecki, and R. Horodecki, Phys. Lett. A 283, 1 (2001).

[6] S. Woronowicz, Rep. Math. Phys. 10, 165 (1976).

[7] M.-D. Choi, "Positive linear maps," (1982).

[8] E. Strmer, Acta Math. 110, 233 (1963).

[9] P. Horodecki, Phys. Lett. A 232, 333 (1997).

[10] B. Kraus, J. I. Cirac, S. Karnas, and M. Lewenstein, Phys. Rev. A 61, 062302 (2000).

[11] P. Horodecki, M. Lewenstein, G. Vidal, and I. Cirac, Phys. Rev. A 62, 032310 (2000).
[12] S. Karnas and M. Lewenstein, Phys. Rev. A 64, 042313 (2001).

[13] S.-M. Fei, X.-H. Gao, X.-H. Wang, Z.-X. Wang, and K. Wu, Phys. Rev. A 68, 022315 (2003).

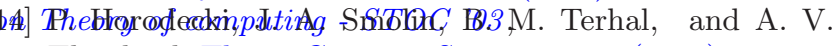
Thapliyal, Theor. Comput. Sci. 292, 589 (2003).

[15] D. Chruściński, J. Jurkowski, and A. Kossakowski, Phys. Rev. A 77, 022113 (2008).

[16] K.-C. Ha, Phys. Rev. A 81, 064101 (2010).

[17] B. Bylicka and D. Chruściński, Phys. Rev. A 81, 062102 (2010).

[18] B. Bylicka, D. Chruściński, and J. Jurkowski, J. Phys. A: Math. Theor. 46, 205303 (2013).

[19] Y. Guo and Hou, J. Phys. A: Math. Theor. 45, 505303 (2012).

[20] X.-Y. Yu and H. Zhao, Int J Theor Phys 54, 292 (2014). 
[21] M. Piani, P. Horodecki, and R. Horodecki, Phys. Rev. Lett. 100, 090502 (2008).

[22] X.-H. Wang and S.-M. Int. J. Quantum Inform. 03, 147 (2005).

[23] L. Gurvits and Phys. Rev. A 66, 062311 (2002).
[24] K.-C. Ha, Phys. Rev. A 87, 024301 (2013).

[25] L. Chen and D. Ž. Đoković, J. Phys. A: Math. Theor. 44, 285303 (2011).

[26] L. Chen and D. Ž. Đoković, J. Phys. A: Math. Theor. 46, 275304 (2013).

[27] A. Sanpera, R. Tarrach, and G. Vidal, Phys. Rev. A 58, 826 (1998). 